\title{
Challenges and opportunities for antimicrobial stewardship in resource-rich and resource-limited countries
}

\begin{abstract}
Introduction: Inappropriate prescription practices, patient and provider knowledge and attitudes, variable availability of diagnostics and surveillance systems, and the unrestricted use of antimicrobials in animals and plants are contributory factors to the global crisis of antimicrobial resistance (AMR).
\end{abstract}

Areas covered: Notwithstanding that interventions to revert AMR should be tailored to the socio-politico-economic landscape, there is global consensus for the implementation and enhancement of antimicrobial stewardship strategies. Yet the implementation of Antimicrobial Stewardship Programs (ASPs) remains relatively limited within healthcare settings and faces complex challenges in resource-limited countries. The current review summarizes the limitations of current ASPs, translation challenges in resource-limited countries, and potential solutions.

Expert opinion: Suboptimal ASP implementation in hospitals is multifactorial. Restriction of antimicrobial use should be informed by risk-benefit analyses, including the potential for substitute prescribing, and displacement of selection pressures. Thresholds in population use of antibiotics above which AMR increases may provide quantitative targets for ASPs. Horizontal and vertical collaborations involving policymakers and the general public are of paramount importance. While impactful prescribing changes require sustained engagement of the public and healthcare professionals, we warn against over-estimating the benefits of behavioral interventions. We advocate for population level stewardship interventions in addition to investment in structural factors that will aid ASP implementation.

Keywords: antimicrobial resistance, antimicrobial stewardship, mainstreaming, regional cooperation, One Health, resource-limited countries, tiered national-level model 


\section{Article highlights:}

- ASPs remain a critical tool in the reversal of AMR within hospital and community settings.

- 'Back-end' strategies are more widely practiced as persuasive and educational initiatives, but 'front-end' strategies and restriction may be more effective.

- Improved diagnostics and surveillance provide opportunities for ensuring empirical and prophylactic therapies are appropriate, and for early rationalization or de-escalation.

- Aggregate prescribing and AMR data can be used to identify the thresholds in population use of specific antimicrobials above which clinical burdens from AMR pathogens increase. This may provide quantitative targets for ASPs and serve as a practical alternative to total restriction of antimicrobials, which is not always feasible or desirable.

- There is evidence suggesting that the indiscriminate use of biocides increases AMR. ASPs should be broadened to include the use of biocides.

- ASPs should be informed by a 'One Health' approach that integrates AMR research relating to human and veterinary health as well as agriculture and the environment.

- A tiered approach, ensuring vertical and horizontal coordination amongst all stakeholders, is key to the implementation of ASPs.

- Economic development is one of the prime determinants of AMR burden and the likely capacity to implement ASPs. International cooperation, technology sharing, and greater investment in AMR research in low- and middle-income countries is needed to adapt ASPs to socio-political, public health and economic challenges, recognizing both the importance of improving access and limiting excess.

- Diffusion of lower cost and point-of-care diagnostics and IT for surveillance, health system strengthening, reductions in out-of-pocket healthcare expenditures, and reducing burdens of infectious disease or associated risk-factors, provide opportunities for ASPs in low- and middle-income countries.

- Behavioral interventions, such as enablement and restriction in particular, are promising tools to modify prescribing trends. 


\section{Introduction}

Lax prescribing standards and the inappropriate use of antimicrobials around the world have contributed to the steady rise of antimicrobial resistance (AMR). It has been confirmed that mortality of patients with bacteremia associated with multidrug resistant infections is significantly higher compared to bacteremia patients where multidrug resistant bacteria was not isolated (40.5\% vs. $28.5 \%, p<0.001)$ (Sirijatuphat et al., 2018). Should the current AMR trend continue, it is estimated that AMR will lead to 10 million deaths every year by 2050 , posing a massive financial and societal problem (O'Neill, 2014). In 2017, the World Bank warned that AMR would exert a drag on global gross domestic product (GDP) from 1.1 to 3.8\% points between then and 2050 (World Bank, 2017). At present, the annual cost of treating antibiotic-resistant infections is already estimated to be 21-34 million US dollars (Infectious Diseases Society of America [IDSA], 2011).

Several interdependent factors spanning across human and animal health, pharmaceuticals, food and agriculture, environment, trade and finance contribute to AMR, making it one of the most complex public health challenges globally. Meeting this challenge is clearly beyond the ability of any single organization or government. Containing and controlling AMR demands international coordinated efforts across the range of sectors mentioned above (World Health Organization [WHO], 2018). The World Health Organization (WHO) instituted a global action plan with the development and implementation of antibiotic stewardship programs (ASP) being one of its key components (WHO, 2015). In 2019, WHO has identified AMR as one of the top ten global health threats $(\mathrm{WHO}, 2019)$. Antimicrobial stewardship continues to play a dominant role in current strategies to tackle AMR (i.e. the United Kingdom's five-year National Action Plan, 2019-2024). As an emerging field, 'antimicrobial stewardship' is still loosely defined as an umbrella of strategies and interventions that seek to measure and improve the use of antibiotics to reverse the rising threat of global AMR (Figure 1). The structure of ASPs differs depending on healthcare and cultural settings, but the core aims of any ASP is to reduce AMR rates, preserve current antibiotics, improve patient outcomes and 
safety, as well as, to reduce the financial costs posed by inappropriate antibiotic prescription. It is important to understand that ASPs are not the elixir to reverse AMR. They should be complemented with regulatory policies and educational measures to control antibiotic use in humans and livestock, the promotion of research and development $(R \& D)$ of new classes of safe and effective antimicrobials and non-antimicrobial alternatives, and, last but not least, infection-control and improved water, sanitation and hygiene (WASH) conditions.

\section{Current antimicrobial stewardship interventions - strengths and limitations}

Successful implementation of ASPs requires the development of targeted strategies to achieve feasible and sustainable outcomes. Within hospital settings, the focus should be on the reduction and streamlining of use of total or specific antimicrobials, the improvement of susceptibility profiles of hospital-borne pathogens, the improvement of clinical markers (such as reduced length of stay) and continuous quality management. Regarding quality assurance, monitoring for unintended harms, such as increasing rates of adverse drug reactions, substitute prescribing, new resistances, worsening clinical outcomes, should be implemented. Measures of direct and indirect benefits or harm of ASPs are listed below.

\section{Measures of direct benefit}

a. Reduction in total antibiotic use (DODs, DDDs, prescriptions per 1000 OBDs or IDs)

b. Reduction in use of targeted (high-risk) antibiotics

c. Minimization in seasonal fluctuations in total or specific prescribing

d. Reduction in sentinel resistant infections (e.g. MRSA, ESBL, multidrug resistant Gram-negative bacteria) or improvements in susceptibility profiles

e. Reduction in acquisitions of colonization

Measures of indirect benefit or harm

a. Change in clinical outcomes 


\section{b. Change in hospital standardized mortality ratios \\ c. Change in infection syndrome-specific mortality \\ d. Change in length of stay and re-admission \\ e. Change in use of non-target antibiotics}

f. Change in adverse drug reactions arising from antibiotic use

These outcomes can be achieved with 'front-end' ASP restrictive strategies where antimicrobials are made available through formulary restrictions and preauthorization. 'Backend' persuasive strategies can also be implemented, where antimicrobial use is reviewed after antimicrobial therapy has been initiated, e.g. prospective audit with intervention and feedback. Back-end strategies are more widely practiced than front-end strategies despite them being more labor-intensive, for the following reasons (Chung, $\mathrm{Wu}, \mathrm{Yeo}$, Chan \& Hsu, 2013): a) higher feasibility and acceptability to clinicians, b) feedback mechanisms and, thus, opportunities for the development of educational and quality enhancement initiatives, c) proved sustained impact on improvement of antimicrobial prescribing practices. As further discussed in section 5.3, it has been shown that enabling strategies enhance the effect of restrictive interventions (Davey et al., 2017).

\subsection{Development of scenario-specific clinical guidelines}

Every country should have a National Action Plan (NAP) addressing the stewardship goals outlined in the WHO Global Action Plan to combat AMR. Such plans should be informed by updated trends in antimicrobial resistance and prevalence of infectious diseases in hospital and community settings. NAPs are an opportunity to identify relevant experts and incorporate their opinions in the development of local treatment algorithms for all frontline healthcare professionals. Multicenter, observational studies have shown that the standardization and streamlining of prescribing guidelines has the acknowledged benefit of a) reducing unnecessary antimicrobial use b) decreasing length of therapy and c) reducing 
prescription costs for hospital-acquired infections (Huijts et al., 2013; Pradelli et al., 2015). On the contrary, a recent meta-analysis revealed that clinical guidelines have no positive effect on resistance rates; perhaps due to short follow-up periods, small sample sizes and poor study designs (Baur et al., 2017). Nonetheless, in resource-limited circumstances adherence to local guidelines can provide initial success which should be followed by resource-demanding core strategies, such as prospective auditing and feedback. The sustained adherence to these guidelines over time may be necessary and intended outcomes should be closely monitored.

\subsection{Education of prescribers}

Guidelines should reach healthcare providers, relevant professional societies and, where appropriate, the general public. Frontline clinicians are educated on antimicrobial prescribing as part of the review of treatment algorithms which can occur while obtaining authorization to use restricted antimicrobials, during formal review and feedback sessions by antimicrobial stewards or through formal didactic sessions. A Cochrane review published in 2013, demonstrated that dissemination via printed educational material or meetings was associated with improved antibiotic use in 5 of 6 studies; the median effect size based on the type of study ranged from $10.6 \%$ to $42.5 \%$. However, no comparative studies have been conducted to determine which educational strategy is most effective. Ultimately, educational strategies are likely most effective when combined with other stewardship strategies such as prospective audit and feedback methods (Davey et al., 2017).

\subsection{Formulary restriction and preauthorization}

Formulary restriction works on the principle that only certain antimicrobials are made available within hospital settings in order to reduce the misuse of broad-spectrum antibiotics. Prior authorization of antimicrobial prescriptions by approved personnel (e.g. medical microbiologists, clinical pharmacists) is another way to directly control antimicrobial misuse and create learning opportunities for prescribers. However, this may lead to prescribers perceiving a loss of autonomy. Antimicrobial prescribing and dispensing policies should be 
reviewed regularly and based on updated AMR trends and good clinical practice. A recent meta-analysis identified 30 studies on this topic. Notwithstanding that most of the studies had a high risk of bias, 26 studies demonstrated that resistance rates for restricted antibiotics were significantly decreased across a wide variety of infective agents and that the overall effect on mortality was non-significant (Schuts et al., 2016). Subsequently, a review of 221 studies confirmed that restriction was independently associated with an increased compliance with antibiotic prescribing policies, with no adverse effects on mortality rates (Davey et al., 2017).

\subsection{Pathogen identification and antibiotic susceptibility testing}

The preferred antibiotic susceptibility testing method remains phenotypic resistance testing. However, this requires a long time to obtain results which inevitably leads to a prolongation of empirical therapy using broad-spectrum antimicrobials (Pulido, García-Quintanilla, MartínPeña, Cisneros \& McConnell, 2013). Novel approaches such as PCR-based techniques, mass spectrometry, microarrays, microfluidics, flow cytometry, isothermal microcalorimetry (IMC), cell lysis-based approaches and whole-genome sequencing have been developed and tested (Pulido, García-Quintanilla, Martín-Peña, Cisneros \& McConnell, 2013; van Belkum \& Dunne, 2013). However, in most cases their sensitivity and specificity compared to reference phenotypic methods still requires validation (Pulido, García-Quintanilla, MartínPeña, Cisneros \& McConnell, 2013). While rapid, accurate and point-of-care pathogen identification to shorten the duration of empirical therapy is advocated as an integral part of ASPs, it is not yet clear whether the benefits of new technologies over existing diagnostics on clinical outcomes and microbial outcomes justifies their huge costs.

\subsection{Digital technology}

The increased use of electronic records and computerized drug ordering systems has created opportunities for the electronic surveillance of prescribing practices and the use of prescribers' guidance. Development of electronic systems in liaison with antimicrobial stewardship teams can enable point-of-care recommendations at entry level to avoid the 
misuse of antimicrobials. Programs may also be equipped to identify allergies, incorrect dosing and mismatches between the antibiotic and susceptibility profiles. Studies have demonstrated a significant positive contribution of electronic systems in the reduction of antimicrobial use, length of hospital stay and costs of antimicrobial prescriptions (Yong, Buising, Cheng \& Thursky, 2010; Filice et al., 2013; Hermsen, VanSchooneveld, Sayles \& Rupp, 2012). Automated time series analysis tools have also been developed to predict susceptibility profiles and evaluate the effect of healthcare interventions on AMR. The predictivity of machine learning algorithms on antimicrobial susceptibility has also been demonstrated (Oonsivilai et al.,2018; WebResist 2.0). Further long-term, multicenter, randomized controlled trials are required to validate effectiveness. Mainstream use of such systems is usually hampered by time and financial requirements for their implementation and maintenance.

\subsection{Pharmacodynamic Dose Optimization}

Pharmacodynamic dosing strategies are aimed at maintaining the concentration of an unbound drug above the minimum inhibitory concentration (MIC) to minimize the emergence of resistance. This is achieved through correlation of pharmacodynamic parameters for individual antimicrobials with efficacy estimates of their achievable tissue concentrations. A recent Cochrane review (Shiu, Wang, Tejani \& Wasdell M, 2013) and multicenter, randomized trial (Dulhunty et al., 2015) in critically ill patients comparing continuous infusions to standard intermittent dosing did not demonstrate superiority in clinical outcomes, although the effect on resistance was not demonstrated. It is therefore important that we emphasize that the effect of sub-optimal dosing on AMR at the population level is not yet known.

\subsection{Antimicrobial cycling}

Antimicrobial cycling is based on the cyclic substitution of a single antimicrobial or an entire class of antibiotics either at the hospital- or unit-level. The rationale behind this technique is that temporary antibiotic removal may minimize the emergence of resistance related to long- 
term use of antimicrobials. A 2014 meta-analysis demonstrated a significant reduction in the incidence of antibiotic-resistant bacteria after this intervention (reduction of 7.2 per 1000 patient-days, $95 \% \mathrm{Cl} 0 \cdot 44-14 \cdot 00 ; \mathrm{p}=0 \cdot 037)$. However, this evidence is weak due to the inclusion of only a small number of studies with short-term follow up periods, which limits the generalizability of the results (Baur et al., 2017; Abel zur Wiesch, Kouyos, Abel, Viechtbauer \& Bonhoeffer, 2014). Indeed, a recent randomized, cross-over trial showed no reduction of AMR in European intensive care units (ICUs) by antibiotic cycling (van Duijn \& Bonten, 2014). Mathematical modelling suggests that either pathogen genotyping or prescribing informed by careful surveillance is superior to antibiotic mixing or cycling (Beardmore, PeñaMiller, Gori, \& Iredell, 2017).

\subsection{Post-prescription review and feedback}

Review and feedback are one of the cornerstones of most ASPs. While the length of antimicrobial treatment remains at the discretion of the treating clinician, a multidisciplinary team will revise the regime after 48-72 hours based on the clinical response and guidelines. Feedback is then provided to prescribers. A multicenter study disclosed that $27.3 \%$ of antibiotic prescribing was "unjustified" and that $66.7 \%$ of clinicians accepted recommendations to amend or discontinue the course of antibiotics. Additionally, it confirmed that a significant reduction in antibiotic usage was observed only in sites with wellestablished ASPs (Cosgrove et al., 2012) as also demonstrated by other studies (Yeo et al., 2012; Newland et al., 2012). This positive effect is likely dependent upon the skills of the multidisciplinary team and the availability of state-of-the-art diagnostics.

\subsection{Periodic Antibiotic Monitoring and Supervision (PAMS)}

PAMS is a real-time antibiotic cycling strategy led by a multidisciplinary antimicrobial stewardship team (made up of two infectious disease physicians, a pharmacist and an infection control nurse). Antibiotic prescribing decisions are supervised to inform periodic categorization of antibiotics into "recommended", "restricted" and "off-supervision" based on AMR data. This strategy has shown significant success (Takesue et al., 2010) which is likely 
a result of real-time monitoring of AMR incidence (Bal, Kumar \& Gould, 2010). Automated and iterative time-series analysis can provide infection-syndrome specific guidance on likely resistance profiles based on temporal trends in resistance, antibiotic prescribing and their relationship (WebResist 2.0).

\subsection{Biomarker-guided diagnostic testing and prescribing}

The reduction in antibiotic prescribing guided by C-reactive protein levels has already been demonstrated and incorporated in the National Institute for Health and Care Excellence (NICE) guidelines for the diagnosis of pneumonia (Quenot et al., 2013; Little et al., 2013). Procalcitonin has been proposed as a potential marker to discriminate between inflammation caused by bacterial versus viral infections (Aabenhus, Jensen, Jørgensen, Hróbjartsson \& Bjerrum, 2014). High strength evidence for procalcitonin-guided discontinuation of antibiotic therapy in adult ICU patients with sepsis, as well as, the initiation of antibiotics for lower respiratory tract infection exists (de Jong et al., 2016). However, most of the evidence emerges from limited studies that have small sample sizes, report modest effect sizes between experimental and control groups, and employ heterogenous cutoff values that hamper us in reaching any tangible conclusion (Andriolo, Andriolo, Salomão \& Atallah, 2017; Bouadma et a., 2010). Studies comparing benefit against established stewardship programs are lacking. Nonetheless, the marginal benefit of biomarker-guided diagnostics, and point-ofcare testing reliant on lay health workers could be greater in resource-limited countries where access to 'expert assessment' is less forthcoming. Indeed, a favorable effect on antibiotic use and clinical outcomes by point-of-care testing has been shown in Tanzanian children with severe infections and in patients attending primary care facilities in Vietnam, Thailand and Myanmar (Keitel et al., 2017; Do et al., 2016; Althaus et al., 2018).

\section{Novel stewardship interventions}


3.1 Thresholds in relationships between population use of specific antibiotics and AMR

Antimicrobial exposure measured at population levels reflect a selection pressure that favors the emergence of multidrug resistant microorganisms. Most ASPs and empirical evidence are based on a 'use it and lose it' principle, in which resistance rises linearly with increasing antibiotic use in the population. However, for several reasons, including the balance between survival advantage and fitness cost of resistance genes, the relationship between antimicrobial use and resistance is more frequently non-linear. Practically, complete restriction of broad-spectrum antibiotics is not always feasible or desirable despite the proven effectiveness of restrictive strategies to reduce AMR rates. For example, overrestriction leads to increased use of alternative antimicrobials leading to new selection pressures or resistance problems (Peterson, 2005). Adverse effects on clinical outcomes are also possible, albeit not proven. At a minimum, over-restriction may lead to a strained relationship between antimicrobial management teams and clinicians.

To date, ASPs have lacked rational quantitative targets for the restriction of specific antibiotic chemical sub-classes or agents within populations. Where increases in the frequency of resistance at the population level are seen only above a critical minimum threshold of antibiotic use, the threshold provides a quantitative target for ASP, optimizing the balance between access to antibiotics for clinical use, and the control of AMR. A recent study using routinely collected data on AMR and population antibiotic use from five European centers, has demonstrated how non-linear time-series analysis can identify critical minimum thresholds in use-resistance relationships (López-Lozano et al., 2019). Importantly, it appears that frequencies of infection with several multidrug resistant pathogens only increase where population-wide use of selecting broad-spectrum antibiotics exceeds a critical level. Such thresholds will provide a quantitative target for ASPs to balance the use of effective antimicrobial therapy and the control of AMR emergence. (López-Lozano et al., 2019). Study authors suggest thresholds should offer "guidance rather than strict limits", but policy options should be determined by the confidence-intervals around thresholds. If strict 
control of resistance is the priority, a conservative approach based on the lower confidence limit around the threshold would be suitable; if excessive restriction is a concern, the point estimate of threshold would be a suitable target. Thresholds are likely to vary across populations and be influenced by host, pathogen, and environmental factors (Lawes et al., 2015; Lawes et al., 2017). Therefore, local data should inform guidance tailored to individual populations and clinical needs. While the use of thresholds is yet to be evaluated in prospective, randomized trials, the current evidence supports its potential to refine ASPs that currently call for avoidance of selected broad-spectrum antibiotics.

\subsection{Biocide stewardship}

We wish to draw the reader's attention to the debate regarding the effect of biocides on the emergence and spread of AMR (Webber et al., 2015; Wand, Bock, Bonney \& Sutton, 2016). This is very important in the context of literature that suggests the superiority of chlorhexidine-based universal decolonization in ICUs over more targeted approaches (Huang et al., 2013) as well as WHO recommendations (Allegranzi et al., 2016) calling for daily skin treatment with chlorhexidine before heart and orthopedic surgery. Research over the last decade has not generated definitive data but there is ample evidence suggesting that biocide exposure may contribute to the selective pressure driving the rise in AMR (Oggioni et al., 2015). Declining susceptibility of Staphylococci, including MRSA, to chlorhexidine has been reported worldwide, prompting concerns over the selection of multidrug resistant Staphylococci by intensive use of chlorhexidine mediated by the resistance gene qacA/B (Otter et al., 2013; Lu et al., 2015; Rondeau et al. 2016). High carriage of genes for chlorhexidine and mupirocin resistance, likely related to the intensive use of these agents in hospitals, has been associated with an increased prevalence of multidrug resistant clones of pathogenic S. epidermidis at the local level and globally (Hijazi et al., 2016; Zamudio, Oggioni, Gould, \& Hijazi, 2019). Biocide resistance to P. aeruginosa has been reported due to mutations in genes, mexR and $\mathrm{nfxB}$, that encode repressors of mexAB-oprM and mexCD-oprJ, respectively (Mc Cay, Ocampo-Sosa \& Fleming, 2010). 
Most concerningly, mechanisms of chlorhexidine resistance in Klebsiella pneumoniae were also associated with cross-resistance to colistin (Buffet-Bataillon, Tattevin, Maillard, Bonnaure-Mallet, \& Jolivet-Gougeon, 2016). We recommend that future epidemiological studies of hospital-acquired multidrug resistant pathogens include a systematic analysis of genes that are associated with a decreased susceptibility to biocides. The findings of this should inform the enhancement of decontamination and infection control policies.

\section{Challenges of stewardship interventions in resource-rich countries}

In most high-income countries there is little evidence of significant changes in increasing secular trends in hospital prescribing of broad-spectrum and last resort antibiotics, such as carbapenems and colistin. This is likely a result of the rising rates of AMR itself (Center for Disease Dynamics, Economics \& Policy, 2015). This AMR spiral has been evidenced in vector-autoregression based time-series models (Toth et al., 2018). However, the escalating use of broad-spectrum antibiotics is not always justified and partly reflects failures in stewardship.

Economic development is one of the main determinants of the likely capacity to implement ASPs. Misuse and abuse of antimicrobials is the primary cause of the rise of AMR in developed countries, while in low and middle-income countries (LMICs) AMR is clearly compounded by additional socio-political, public health and economic aspects as described in section 5 (Laxminarayan, Sridhar, Blaser, Wang \& Woolhouse, 2016; Resistance Map. Center for Disease Dynamics, Economics \& Policy, 2017; Klein et al., 2018). The following key challenges associated with the implementation of ASPs in resource-rich countries have been identified:

1. Tailoring of antimicrobial prescribing guidelines to the needs of individual health boards or hospitals.

2. Effective coordination among members of the antimicrobial stewardship teams.

3. Auditing of adherence to recommendations and identifying areas for improvement. 
4. Diagnostic uncertainty and fear of poor clinical outcomes leading to unnecessary antibiotic prescribing, prolonged use of broad-spectrum antibiotics, use of redundant combinations of drugs and failure to de-escalate therapy.

5. Understanding the role of psychosocial determinants, such as the patients' expectations and the health professionals' perception of those expectations.

6. Understanding the role of clinical team dynamics on compliance with ASPs.

7. Factoring the role of reimbursement systems of national health services and corporate interests of the pharmaceutical industry.

Intensive use of antibiotics contributes to AMR not only when used for definitive treatment, but also within prophylactic as well as empirical regimens.

\subsection{Risk-benefit of antimicrobial stewardship vs. antimicrobial prophylaxis}

Compliance with evidence-based approaches is advocated to avoid prescribing practices that hamper the success of ASPs. Indications for antimicrobial prophylaxis have historically included prevention of surgical site infections (SSIs), hospital-acquired and aspiration pneumonia, recurrent urinary tract infections, asymptomatic bacteriuria and recurrent cystitis. However, the evidence only supports the use of prophylaxis in the prevention of SSIs. Antibiotic prophylaxis in the context of hospital-acquired pneumonia and aspiration pneumonia has consistently shown no effect on mortality rates (Dragan et al., 2018). On the contrary, there is a concern that it may cause microbial imbalances that enhance the risk for infection by multidrug resistant organisms. Similarly, a Cochrane study that included five clinical trials showed limited evidence to support the benefits of antibiotic prophylaxis in recurrent urinary tract infections (Niel-Weise, van den Broek, da Silva \& Silva, 2012). A recent randomized controlled trial showed a decreased recurrence of urinary tract infections in high-risk patients but confirmed an increase in multidrug resistant bacteria in urine and fecal samples (Fisher et al., 2018). Equally, a meta-analysis to assess prophylaxis in recurrent urinary tract infections among children showed an increased risk of multidrug 
resistant infections (Selekman et al., 2018). Accordingly, the latest European Association of Urology (EAU) guidelines recommend using continuous antibiotic prophylaxis as a last resort in cases where alternative strategies have been exhausted (Grabe et al., 2015).

\subsection{Antimicrobial stewardship and empirical prescribing}

Empirical antimicrobial therapy is a critical tool in the early treatment of acute infections as well as in the prevention of sepsis and its life-threatening sequelae (Dellinger et al., 2013; National Confidential Enquiry into Patient Outcome and Death [NCEPOD], 2015). Empirical prescribing guidelines call for broad-spectrum antibiotics in certain scenarios, for example in cases of hospital-acquired pneumonia, or combinations of antibiotics for cases of suspected sepsis. While advocacy for prompt and effective management of sepsis is justified, the risks of overuse of antibiotics arising from sepsis policies are little explored. Moreover, there is growing evidence that inappropriate antibiotic treatment leads to worse outcomes after empirical therapy, suggesting that survival from sepsis depends on the control of AMR. Reconciliation of prompt management of sepsis and the control of AMR requires careful clinical algorithms as well as rapid or point-of-care testing that can accurately identify between serious infections.

More simply, it is critical to consider the choice of antibiotic, mode of delivery, an intravenous (IV) switch to an oral route and duration of the antibiotic course, the awareness of which should extend to all those directly involved in the prevention and treatment of sepsis, including emergency physicians, intensivists and surgeons. Infectious diseases doctors, medical microbiologists and clinical pharmacists can provide invaluable expertise to inform these aspects. A multicenter, prospective, observational, 72-hour snapshot study involving 67 intensive care units from 32 hospitals in the United States showed alarming findings regarding prolonged empirical antibiotic treatment. Out of 660 antibiotics that were empirically prescribed to 364 patients, 333 were continued for at least 72 hours in the absence of a confirmed infection. Suspected pneumonia accounted for approximately $60 \%$ of empirical prescribing and the most frequently prescribed antibiotics were vancomycin and 
piperacillin/tazobactam (Thomas et al, 2015). A UK study, focused on prescribing in primary care, also showed that the duration of antibiotic treatments exceeded prescribing guidelines (Pouwels et al., 2019). Further, multiple studies suggest shorter regimens can be equivalent in most clinical scenarios. In fact, it has been demonstrated that the duration of antibiotic treatment directly relates to AMR indicating that the indiscriminate advice of 'completing course' is not justified (Teshome, Vouri, Hampton, Kollef,\& Micek, 2019).

Taken together, these findings stress the importance of reassessment and de-escalation of antimicrobial therapy based on clinical progress, microbiology culture and susceptibility testing. More specifically, it highlights the need for: a) a review of antimicrobial prescriptions at 48-72 hours where de-escalation to a narrow-spectrum agent or escalation in line with susceptibility results is carried out; b) the use of electronic prescribing systems to remind prescribers to review antimicrobial therapy after $48-72$ hours and alert them to potential mismatches with microbiological culture results (Garnacho-Montero, Escoresca-Ortega \& Fernández-Delgado, 2015; Masterton, 2011). De-escalation is safe. Observational study data suggest that de-escalation may reduce hospital stay and even mortality in pneumonia (Paul, Dickstein \& Raz-Pasteur, 2016; Ambaras Khan \& Aziz, 2018) while a randomized controlled trial showed that shortened antibiotic use guided by procalcitonin reduced mortality (de Jong et al., 2016). Clearly, organizations should ensure prescribers have ready access to the latest evidence-based guidelines. These guidelines must be reviewed periodically in response to local antibiograms and in line with the most up to date research.

\section{Hurdles to global implementation of antimicrobial stewardship programs}

The control of AMR is a global responsibility and is clearly beyond the scope of a single government, department or independent organization, hence the commitment of the WHO in tackling AMR. Five factors have been identified as roadblocks to successful implementation of ASPs in many countries (Figure 2) (Tiong, Loo \& Mai, 2016): i) awareness and political 
will, ii) finance, iii) coordination, iv) monitoring and v) data and technical capacity. Nonetheless, it is acknowledged that national income and adequate financial capacity is a pre-requisite to successful implementation of ASPs. Problems associated with suboptimal ASP implementation in high-income countries, such as the lack of political support and excessive bureaucracy, is compounded in LMICs by the general lack of dedicated funding, poor infrastructure and workforce shortages.

\section{i. $\quad$ Awareness and political will:}

Governments should take ownership of ASP implementation and ensure that it is adequately resourced. No substantial progress can be made to reverse AMR unless it is made a national priority and the leadership needed to drive ASP actions are taken at the government-level. Success of ASPs is also dependent on awareness and engagement amongst the general public.

ii. Finance:

Sustained investment and funds are required to build systems and support needed for the implementation of ASPs. Shortage of public funding is usually a reflection of the fact that ASP implementation is not seen as a development priority nor is it embedded in government planning or budgeting cycles. Governments should also institute strategies to incentivize contributions from the private sector. Joint public-private investments should follow a 'One Health' approach, extending the principles of ASPs beyond human health to include animal, plant and environmental health sectors.

iii. Coordination across sectors and stakeholders:

Coordinated actions spanning across human, animal and environmental health requires horizontal collaboration across all stakeholders through diverse activities, for example knowledge-sharing platforms and multi-stakeholder forums, supported by a tiered approach encompassing vertical collaboration from central policymakers to frontline health practitioners. Generally, the effectiveness of coordination within sectors is partly dependent on the quality of education and training in networking, 
communication and governance. In resource-limited countries cross-sectorial coordination is hampered by the lack of (or poorly resourced) governance policies and mechanisms to bridge different sectors. Limited regulation or influence over the private sector can also compromise effective cross-sectorial coordination.

iv. Monitoring and evaluation systems:

A robust monitoring and evaluation framework is essential in identifying targets, tracking longitudinal progress and ensuring accountability. However, this is clearly dependent on political stability and adequate funding specifically dedicated to these initiatives.

v. Data and technical capacity:

Relatively few countries have local and national registries on antimicrobial use and AMR that can be used to track the effect of ASPs. Such registries provide essential evidence to influence all those involved in NAPs on AMR.

\subsection{Socio-politico-economic factors}

A recent report suggests that a range of social, political and economic factors contribute directly to AMR globally (Collignon, Beggs, Walsh, Gandra \& Laxminarayan, 2018). According to this review of data for 103 countries, aspects of "good governance" (lower corruption, political stability, rule of law and lower crime), quality of infrastructure (sanitation, safe water, internet accessibility, urbanization, and access to electricity) and educational attainment were negatively correlated with AMR indices. Interestingly, at a national level, 'official' measures of antibiotic use were not associated with AMR. This surprising finding points to the concerning possibility that a large proportion of antibiotics is sourced from outside the public sector and even outside formal healthcare. Another study found a positive association between rising $\mathrm{AMR}$ and access to poor quality antibiotics and falsified products (Ozawa et al., 2018). In terms of the effect of management of healthcare systems, higher investment in public healthcare services were associated with lower AMR while higher ratios 
of private to public health expenditure correlated with higher AMR indices (Collignon, Beggs, Walsh, Gandra \& Laxminarayan, 2018). Previously, it has been shown that out-of-pocket expenditure in the public sector correlated positively with AMR by encouraging patients to seek out private care (Alsan et al., 2015) where commercial incentives to over-prescribe are enhanced (section 4.3).

\subsection{Commercialization and corporate-driven antimicrobial prescribing}

The risk-adjusted net profit value of antibiotics is three-fold lower compared to an anti-cancer drug, seven times lower than neurological drugs and more than ten times lower than musculoskeletal drugs (Morel \& Mossialos, 2010). Arguably, commercial profits are likely to be adversely affected by any initiative aimed at controlling acute infections. This is the main reason for the notoriously low interest of the pharmaceutical industry in the development of new technologies to control infections. As a result, the onus of combatting AMR has fallen entirely on governmental agencies. Collaborative programs between public and private sector, by analogy with those established to tackle the HIV/AIDS crisis, are urgently needed to foster the development of new treatments and concomitant implementation of ASPs.

The pharmaceutical industry influences drug prescription practices either directly or indirectly. For every newly discovered drug, pharmaceutical firms are given temporary monopolistic power through a patent to recover research and development costs. The monopoly and restricted availability of a certain antimicrobial can result in under-prescribing, which increases infection levels that will in turn increase the demand as well as the monetary value of the antimicrobial. Further, profit-driven monopolies will usually neglect longitudinal data on the efficacy of the new drug and epidemiology of infections that may compromise revenue (Kelly, Zoubiane, Walsh, Ward \& Goossens, 2016).

At the end of the patent period market, competition increases overall market size of the product which can drive over-prescribing and impact negatively on efforts to control AMR (Herrmann, 2010). This profit-driven approach undermines ASPs at many levels with detrimental effects reaching the zenith in market-based healthcare systems. Further 
research is required to understand the impact of the market on AMR trends. Findings should inform the development of policies that factor in the management of corporate profits while prioritizing NAPs for AMR.

\subsection{Animal and environmental antimicrobial resistance}

The boost of epidemiological, genetic and mechanistic research relating to AMR in animal and environmental health, as well as its integration with AMR research directly relating to human health, has been advocated at a global level for quite some time (O'Neill, 2014; One Health Basics, 2018). Unfortunately, a systematic observational study showed that only $3 \%$ of current AMR research funding supports projects relating to environmental AMR (Kelly, Zoubiane, Walsh, Ward \& Goossens, 2016). Cost-effective environmental surveillance of AMR itself is an area in significant need of development. While the number of studies in animal health-related research has surged in the last few years, sustained and systematic national surveillance of AMR in the animal sector is lacking. This makes the integration of animal data with AMR surveillance data in hospitals and the community difficult.

Based on an FDA report, the total amount of antibiotics sold in the United States for food production animals exceeded that sold for human consumption (CDC, 2012). Use of antimicrobials as growth promotors for food production animals, including in aquaculture, is an area that requires urgent attention as this practice is currently only banned in Europe. The intensive use of antibiotics in the farming industry has contributed to the rise of AMR in food production animals that, in turn, has spread to humans through the food supply (Collignon, Powers, Chiller, Aidara-Kane \& Aarestrup, 2009). A recent meta-analysis confirmed that restriction of antibiotic use in food production animals is associated with a reduction in AMR in these animals, while evidence of the impact on the general human population is more limited and mostly related to direct exposure to food production animals (Tang et al., 2017). Future molecular epidemiological studies should also evaluate the longterm effect on the human resistome (Vanderhaeghen \& Dewulf, 2017). In any event, restriction measures should be tailored to the national economic landscape in order to 
minimize the impact on resource-limited farming and food production systems already struggling with food security (Littmann, Buyx \& Cars, 2015). Restriction should also be accompanied by the enhancement of husbandry and housing practices, improved biosecurity and infection control measures, including vaccination, all of which will reduce unnecessary use of antimicrobials. The key barriers for successful implementation of ASPs within veterinary medicine are governance structures, client expectations and market competition, cost of microbiological testing and lack of access to education, training and resources (Parsonage et al., 2017). While some of the antimicrobial stewardship principles which are meant for humans may also be applicable to tackling AMR in the animal sector, it is likely that a significant reduction of antimicrobial use in both the animal and environmental sectors will ultimately be driven by the development of alternatives to antibiotics. Indeed, the development of quality and affordable animal vaccines as well as rapid and affordable pointof-care tests to guide empirical use of antimicrobials in animals are two priority areas (Magouras, Carmo, Stark \& Schupbach-Regula, 2017).

An area that has received very little attention is in the management of disposed antimicrobials. Antimicrobials are released into the environment on a massive scale either by improper disposal of antibiotics, unregulated dissemination of agricultural waste (Berendsen, Wegh, Memelink, Zuidema, \& Stolker, 2015) or inadequate treatment of wastewater (Chen, Zhang, Ying, Zhou, \& Jones, 2015). The cumulative burden of antimicrobials that re-enters the ecosystem will likely exert selection pressure on environmental microbial communities, and in turn contribute to the rise in AMR. This may be compounded by the release of heavy metals and biocides (Frontiers report 2018/19), notwithstanding that the concentrations of antimicrobial agents found in surface waters and soil environments are lower than levels used in clinical or industrial settings. Stringent measures should be taken to further regulate the disposal of farming, industrial and household waste containing antimicrobials. Horizontal coordination between the public and policymakers is essential to root out unsafe antimicrobial disposal and develop sustainable mitigation technologies. However, 
implementation of strategies to reduce or remove antibiotics from waste streams are often hampered by financial barriers. Further, environmental exposure to multidrug resistant pathogens and antibiotics in resource-limited countries is compounded by inadequate sanitation perpetuated by socio-political obstacles, e.g. rapidly emerging urban slums which are deprived of sanitation to by-pass the provision obligations associated with the 'formal settlement' status. Ultimately, sanitation initiatives to control AMR should be integrated with the wider WASH agenda.

\section{Strategies to increase global ASP implementation}

\subsection{Tiered national-level model}

To date most implementation strategies have been directed at high-income countries that already have robust regulatory frameworks and well-functioning healthcare systems. As a matter of priority, the focus of ASPs should be broadened to include LMICs that are hot spots for AMR. A non-exhaustive list of challenges associated with AMR control in LMICs include poor water quality and sanitation, overcrowded housing, poor infection control practices, under-resourced healthcare systems, lack of prescribing policies and guidelines, suboptimal infectious diseases and clinical pharmacy training programs, and weak data management systems. "Tiered national-level antibiotic stewardship activities" laid down by CDC may be a pragmatic tool to guide the introduction of ASPs in LMICs (CDC. The Core Elements of Human Antibiotic Stewardship Programs in Resource -Limited Settings: National and Hospital Levels, 2018). According to this model, ASP activities are tiered into A) basic: not requiring dedicated resources and primarily reliant on existing personnel and technical expertise, B) intermediate: requiring dedicated staff for ASP planning and implementation as well as some level of estate investment, C) advanced: requiring the establishment of a formal and holistic program with trained staff and dedicated infrastructure as discussed in previous sections. 
Basic: A multi-sectorial decision-making ASP body sets a NAP on AMR aligned with the stewardship goals of the WHO Global Action Plan but tailored to local needs and availability of resources. Equally, at the operational level, representatives from a range of relevant sectors - ministries, civil society and healthcare - are responsible for implementing the ASP activities within the NAP in a coordinated fashion. ASPs should include public engagement initiatives to raise awareness of the potentially catastrophic effects of the misuse of antibiotics.

Intermediate: In additional to the above, this model should include an institution of registries on epidemiological data on community- and hospital-acquired infections to inform the development of evidence-based treatment guidelines. Rural and primary healthcare centers should have easy access to appropriate formularies for ACCESS antibiotics, as defined by the WHO essential medicine list, while RESERVE antibiotics may be limited to hospitals. Investment in enhancing the efficiency of diagnostic testing should also be integral at this tier (WHO updates Essential Medicines List with new advice on use of antibiotics, and adds medicines for hepatitis C, HIV, tuberculosis and cancer, 2017).

For both models we strongly advocate that public sector initiatives are associated with stringent regulations of the private and informal sectors.

\subsection{Mainstreaming and regional cooperation}

Mainstreaming initiatives are based on optimizing the uptake of existing ASPs and enhancing the programs themselves rather than de novo initiatives. In a mainstreamed approach, the ASP should be anchored to existing strategic plans concerning human, animal and environmental health. Cross-sectorial coordination within mainstreaming initiatives is facilitated through an initial focus on priority sectors and the gradual incorporation of additional sectors horizontally and vertically.

The first step in mainstreaming is to map national activities and identify potential entry points. To ensure full participation, transparency and legitimacy, such mapping should cover 
both governmental and non-government activities. Decision tools, tailored to the disease burden and socioeconomic status, can then be used to identify the activities that are easiest to scale up, most cost-effective and have the greatest impact on AMR and infection control outcomes.

Analogous to climate change agreements, international cooperation is a need of the hour, particularly in countries divided politically but united by common cultural, social and economic backgrounds. Examples include "the European One Health Action Plan against AMR" and "the ASEAN regional strategy on AMR". Increased regional cooperation helps to tackle the cross-border threat of AMR, ensuring that inaction in one area does not undermine progress in others. It strengthens solidarity and opens the door for knowledge exchange and learning. Principles of regional cooperation include:

a) Common funding mechanisms to mobilize resources within regions.

b) Joint procurement programs to help small or resource-poor countries to access a sustainable (and cheaper) supply of good-quality antimicrobials at reduced transaction costs.

c) Shared data platforms to collect and analyze antibiotic use and AMR data in human and animal populations.

d) Shared infrastructure e.g. regional laboratory facilities, to support cross-border AMR monitoring.

e) Shared regulatory and legal frameworks to coordinate and implement crossregional legislation and guidelines.

f) Joint research networks and training programs.

g) Joint awareness campaigns to increase the understanding of AMR among stakeholders throughout a region. Multi-stakeholder networks and forums for knowledge exchange can help countries learn from each other, share ideas, build consensus, compare progress and accelerate national work. 
The implementation of mainstreaming and regional cooperation is clearly dependent on strong political commitment and adequate funding to the following areas: i) surveillance of AMR; ii) surveillance of antimicrobial use; iii) development and implementation of national infection control standard treatment guidelines; iv) operational research on antimicrobial usage, environmental surveillance and antibiotic susceptibility testing methodology; and v) antimicrobial stewardship education of healthcare workers and awareness amongst the general public.

\subsection{Behavioral interventions}

Alongside strategies tackling social, political and economic barriers, as described above, it has been suggested that interventions aimed at modifying prescribing behavior at the individual level may have a significant impact on AMR control. However, it is not clear which behavioral technique can effectively improve prescribers' attitudes towards antibiotic prescribing. A Cochrane review that included 221 studies demonstrated, with high-certainty evidence, that both enablement and restriction were independently associated with increased compliance with antibiotic prescribing guidelines (Davey et al., 2017). It was also shown that such interventions reduced the duration of antibiotic treatment and length of hospital stay. The same report showed that feedback strategies were equally effective, but the number of relevant studies was limited. Nonetheless, the authors raised concerns that feedback and restriction interventions can lead to delays in treatment and a breakdown of trust between infection specialists and clinical teams. More research studies are needed to assess the unwanted effects of restrictive strategies. Studies exploring action planning and goal setting were too few to infer any conclusions with regards to the benefit of these behavioral change techniques.

Future research should further establish which behavioral change strategy will likely facilitate ASP implementation with consideration to additional challenges faced by resource-limited countries (Davey et al., 2017; Rzewuska et al., 2019). 


\subsection{Public awareness}

Implementation of ASPs should transcend beyond healthcare professionals to involve active participation of the general public. Systematic reviews have demonstrated that joint involvement of physicians and patients facilitates the reduction of unnecessary antibiotic use (Arnold \& Straus,2005; Ranji, Steinman, Shojania \& Gonzales, 2008). Educational campaigns on the appropriate use of antimicrobials using written material and mass media have been shown to bring a positive behavioral change amongst patients and the general public (C. Lee, J. Lee, Kang, Jeong \& S. Lee, 2015). The individual impact of various public campaigns in Europe between 1997 and 2007 has been estimated to be equivalent to a 6.5$28.3 \%$ drop in the mean level of overall antibiotic use (Filippini, Ortiz \& Masiero, 2013), although arguably it is not possible to demonstrate its independent effect from other components of ASPs. The potential benefit of embedding antimicrobial stewardship principles in school educational programs has been reported in relatively small studies (C. Lee, J. Lee, Kang, Jeong, \& S. Lee, 2015), but the long-term effect of these initiatives is yet to be evaluated. Clearly AMR campaigning should go hand in hand with awareness of key basic infection control practices (Curtis et al., 2011). Unfortunately, recent reports suggest limited AMR awareness amongst the general public. For example, a lack of awareness that antibiotics fail to relieve viral infections and that prescribed medications should not be shared or hoarded, have been reported (Jamhour, El-Kheir, Salameh, Hanna, \& Mansour, 2017). While this data confirms the importance of public campaigning, we wish to stress that increased awareness of AMR does not necessarily translate into behavioral changes that will facilitate the implementation of ASPs.

\section{Conclusion}

Unfettered use of antimicrobials in humans, animals, plants and the environment has led to an increase in global AMR rates. Antimicrobial stewardship could potentially contribute to the reversal of current AMR trends. ASPs should be tailored to the socioeconomic status of each 
country, and efforts to identify social, political and economic factors contributing to AMR should continue. ASPs must be implemented within both hospital and community settings and be driven by cross-sectorial coordination horizontally and vertically. There is currently no definitive evidence to demonstrate the superiority of a single antimicrobial stewardship strategy. In the interim, monitoring of the adherence to evidence-based clinical guidelines should be practiced. We also strongly advocate that ASP models are informed by population level thresholds of antibiotic use. The development of point-of-care diagnostics and rapid antimicrobial susceptibility testing to minimize empirical prescribing of broad-spectrum antimicrobials should be a priority. Consideration must be given to broadening the scope of current ASPs to include biocide use in hospitals. Global implementation and success of ASPs rests on five pillars - governmental commitment, multi-sectorial collaboration, AMR surveillance, funding and monitoring-feedback systems to track progress. The tiered national-level model as well as mainstreaming and regional cooperation may facilitate the global implementation of ASPs, while behavioral interventions to modify prescribing trends also merit attention. Research relating to AMR in animal and environmental health as well as its integration with human data requires a significant boost in order to pursue the 'One Health' approach. Enhanced investment by both the public and private sectors in new technologies to control infections and AMR should be a priority. Joint public-private sector initiatives may facilitate the sustained and concrete commitment of the pharmaceutical industry. 


\section{Expert Opinion}

AMR is a global health threat that spans the entire socioeconomic spectrum and remains one of the top ten WHO priorities. To tackle this complex issue, all contributing factors should ideally be addressed within NAPs for AMR, resources permitting. ASPs can rationalize the use of antibiotics and make a significant contribution to tackling AMR but they should be tailored to the needs and financial capacity of individual countries. On the other hand, the onus of identifying the factors that contribute to AMR rates and customizing the general principles of ASPs should fall on individual countries or preferably regions in the spirit of regional cooperation. The availability of resources and the strength of infrastructure are two main determinants that should shape ASPs. Ultimately, the successful implementation of ASPs depends on the selection of an appropriate strategy. Notwithstanding that high-quality evidence to demonstrate the superiority of a single antimicrobial stewardship strategy over others is lacking, any ASPs should include, as a bare minimum, the evaluation of adherence to evidence-based clinical guidelines for antimicrobial use and the use of feedback to enhance compliance. We strongly advocate ASP models informed by population level thresholds of antibiotic use over arbitrary total restrictions of broad-spectrum antibiotics. Importantly, the impact of ASPs should be monitored at timely intervals and their content revised based on the current epidemiology of community- and hospital-acquired infections and AMR data.

The development of point-of-care diagnostic and rapid antimicrobial susceptibility testing to minimize empirical prescribing of broad-spectrum antimicrobials should be a priority. Consideration must be given to broadening the scope of current ASPs to include biocide use in hospitals. While more research is required to fully assess the impact of biocide use on AMR trends, based on current evidence we suggest that indiscriminate use of biocides should be avoided.

AMR is clearly a multi-sectorial problem, hence the lack of cross-sectorial coordination (vertical and horizontal) and accountability are two major obstacles to the achievement of the 
desired ASP outcomes. Increased clarity and transparency of the roles of representatives from different sectors will partly tackle these two issues. Global implementation and success of ASPs rests on five pillars - governmental commitment, multi-sectorial collaboration, AMR surveillance, funding and continuous monitoring. The tiered national-level model as well as mainstreaming and regional cooperation may facilitate global implementation of ASPs - in particular ASP adoption and implementation in resource-limited countries.

The remit of ASPs should span across humans, animals, plants and the environment in perusal of the 'One Health' approach advocated by all AMR NAPs. However, more research is required to enhance our understanding of AMR in all these areas, specifically as to how they interact with one another. In the interim, policies to minimize unnecessary or subclinical doses of antimicrobials in animals and plants should be enforced.

Enhanced investment in the discovery of new antibiotics and other cost-effective alternatives by both the public and private sector should be a priority. We suggest that access to antimicrobials should be driven by public health evidence and should not be influenced by the commercial interests of the pharmaceutical industry.

Behavioral interventions to modify prescribing trends are a promising area but more work is required to identify specific techniques that will likely contribute to changing antimicrobial prescribing behavior. The focus on patient attitude is also critical, thus public engagement initiatives to promote responsible use of antibiotics as well as fundamental infection control and sanitation practices are important. While impactful changes in antimicrobial prescribing practices will clearly require sustained engagement and commitment of the public and healthcare professionals, we warn against over-estimating the potential benefit of behavioral interventions. We note a general misalignment between personal behavior and commitment to societal impact and, on this basis, strongly advocate population level stewardship interventions in association with an investment in structural factors that will aid the implementation of ASPs. 


\section{References}

Aabenhus, R., Jensen, J. U., Jorgensen, K. J., Hrobjartsson, A., \& Bjerrum, L. (2014). Biomarkers as point-of-care tests to guide prescription of antibiotics in patients with acute respiratory infections in primary care. The Cochrane Database of Systematic Reviews, 11 ,CD010130. doi: 10.1002/14651858.CD010130.pub2

Abel zur Wiesch, P., Kouyos, R., Abel, S., Viechtbauer, W., \& Bonhoeffer, S. (2014). Cycling empirical antibiotic therapy in hospitals: Meta-analysis and models. PLoS Pathogens, 10(6), e1004225. doi: 10.1371/journal.ppat.1004225

Allegranzi, B., Zayed, B., Bischoff, P., Kubilay, N. Z., de Jonge, S., de Vries, F., ... WHO Guidelines Development Group. (2016). New WHO recommendations on intraoperative and postoperative measures for surgical site infection prevention: An evidence-based global perspective. The Lancet.Infectious Diseases, 16(12), e288-e303. doi:

10.1016/S1473-3099(16)30402-9

Alsan, M., Schoemaker, L., Eggleston, K., Kammili, N., Kolli, P., \& Bhattacharya, J. (2015). Out-of-pocket health expenditures and antimicrobial resistance in low-income and middle-income countries: an economic analysis. The Lancet. Infectious diseases, 15(10), 1203-1210. doi:10.1016/S1473-3099(15)00149-8

Althaus, T., Greer, R. C., Swe, M., Cohen, J., Tun, N. N., Heaton, J., ... Lubell, Y. (2018). Effect of point-of-care C-reactive protein testing on antibiotic prescription in febrile patients attending primary care in Thailand and Myanmar: an open-label, randomised, controlled trial. The Lancet. Global health, 7(1), e119-e131. doi:10.1016/S2214$109 \times(18) 30444-3$

Ambaras Khan, R., \& Aziz, Z. (2018). Antibiotic de-escalation in patients with pneumonia in the intensive care unit: A systematic review and meta-analysis. International Journal of Clinical Practice, 72(10), e13245. doi: 10.1111/ijcp.13245 
Andriolo, B. N., Andriolo, R. B., Salomao, R., \& Atallah, A. N. (2017). Effectiveness and safety of procalcitonin evaluation for reducing mortality in adults with sepsis, severe sepsis or septic shock. The Cochrane Database of Systematic Reviews, 1, CD010959. doi: 10.1002/14651858.CD010959.pub2

Arnold, S. R., \& Straus, S. E. (2005). Interventions to improve antibiotic prescribing practices in ambulatory care. The Cochrane Database of Systematic Reviews, 4, CD003539. doi: 10.1002/14651858.CD003539.pub2

Bal, A. M., Kumar, A., \& Gould, I. M. (2010). Antibiotic heterogeneity: From concept to practice. Annals of the New York Academy of Sciences, 1213, 81-91. doi: 10.1111/j.1749-6632.2010.05867.x

Baur, D., Gladstone, B. P., Burkert, F., Carrara, E., Foschi, F., Döbele, S., \& Tacconelli, E. (2017). Effect of antibiotic stewardship on the incidence of infection and colonisation with antibiotic-resistant bacteria and clostridium difficile infection: A systematic review and meta-analysis. The Lancet.Infectious Diseases, 17(9), 990-1001. doi: $10.1016 / S 1473-3099(17) 30325-0$

Beardmore, R. E., Peña-Miller, R., Gori, F., \& Iredell, J. (2017). Antibiotic Cycling and Antibiotic Mixing: Which One Best Mitigates Antibiotic Resistance? Molecular biology and evolution, 34(4), 802-817. doi:10.1093/molbev/msw292

Berendsen, B. J., Wegh, R. S., Memelink, J., Zuidema, T., \& Stolker, L. A. (2015). The analysis of animal faeces as a tool to monitor antibiotic usage. Talanta, 132, 258-268. doi: 10.1016/j.talanta.2014.09.022

Bouadma, L., Luyt, C. E., Tubach, F., Cracco, C., Alvarez, A., Schwebel, C.,... PRORATA trial group. (2010). Use of procalcitonin to reduce patients' exposure to antibiotics in intensive care units (PRORATA trial): A multicentre randomised controlled trial. Lancet, 375(9713), 463-474. doi: 10.1016/S0140-6736(09)61879-1 
Buffet-Bataillon, S., Tattevin, P., Maillard, J. Y., Bonnaure-Mallet, M., \& Jolivet-Gougeon, A. (2016). Efflux pump induction by quaternary ammonium compounds and fluoroquinolone resistance in bacteria. Future Microbiology, 11(1), 81-92. doi: 10.2217/fmb.15.131

CDC. The Core Elements of Human Antibiotic Stewardship Programs in Resource-Limited Settings: National and Hospital Levels. Atlanta, GA:US Department of Health and Human Services, CDC; 2018. Retrieved from: https://www.cdc.gov/antibioticuse/healthcare/implementation.html

Center for Disease Dynamics, Economics \& Policy (2015) State of the World's Antibiotics, 2015. CDDEP: Washington, D.C. Retrieved from: https://cddep.org/wpcontent/uploads/2017/06/swa edits 9.16.pdf

Chen, C. E., Zhang, H., Ying, G. G., Zhou, L. J., \& Jones, K. C. (2015). Passive sampling: A cost-effective method for understanding antibiotic fate, behaviour and impact. Environment International, 85, 284-291. doi: 10.1016/j.envint.2015.10.001

Chung, G. W., Wu, J. E., Yeo, C. L., Chan, D., \& Hsu, L. Y. (2013). Antimicrobial stewardship: A review of prospective audit and feedback systems and an objective evaluation of outcomes. Virulence, 4(2), 151-157. doi: 10.4161/viru.21626

Coast, J., Smith, R. D., \& Millar, M. R. (1998). An economic perspective on policy to reduce antimicrobial resistance. Social Science \& Medicine (1982), 46(1), 29-38. doi: $10.1016 / S 0277-9536(97) 00132-9$

Collignon, P., Beggs, J. J., Walsh, T. R., Gandra, S., \& Laxminarayan, R. (2018). Anthropological and socioeconomic factors contributing to global antimicrobial resistance: A univariate and multivariable analysis. The Lancet.Planetary Health, 2(9), e398-e405. doi: 10.1016/S2542-5196(18)30186-4 
Collignon, P., Powers, J. H., Chiller, T. M., Aidara-Kane, A., \& Aarestrup, F. M. (2009). World health organization ranking of antimicrobials according to their importance in human medicine: A critical step for developing risk management strategies for the use of antimicrobials in food production animals. Clinical Infectious Diseases : An Official Publication of the Infectious Diseases Society of America, 49(1), 132-141. doi: $10.1086 / 599374$

Cosgrove, S. E., Seo, S. K., Bolon, M. K., Sepkowitz, K. A., Climo, M. W., Diekema, D. J., ... CDC Prevention Epicenter Program. (2012). Evaluation of postprescription review and feedback as a method of promoting rational antimicrobial use: A multicenter intervention. Infection Control and Hospital Epidemiology, 33(4), 374-380. doi: $10.1086 / 664771$

Curtis, V., Schmidt, W., Luby, S., Florez, R., Toure, O., \& Biran, A. (2011). Hygiene: New hopes, new horizons. The Lancet.Infectious Diseases, 11(4), 312-321. doi: $10.1016 / S 1473-3099(10) 70224-3$

Davey, P., Marwick, C. A., Scott, C. L., Charani, E., McNeil, K., Brown, E.,..., Michie S. (2017). Interventions to improve antibiotic prescribing practices for hospital inpatients. The Cochrane Database of Systematic Reviews, 2, CD003543. doi: 10.1002/14651858.CD003543.pub4

de Jong, E., van Oers, J. A., Beishuizen, A., Vos, P., Vermeijden, W. J., Haas, L. E., ... de Lange, D. W. (2016). Efficacy and safety of procalcitonin guidance in reducing the duration of antibiotic treatment in critically ill patients: A randomised, controlled, openlabel trial. The Lancet.Infectious Diseases, 16(7), 819-827. doi: 10.1016/S14733099(16)00053-0

Dellinger, R. P., Levy, M. M., Rhodes, A., Annane, D., Gerlach, H., Opal, S. M.,... Surviving Sepsis Campaign Guidelines Committee including The Pediatric Subgroup. (2013). Surviving sepsis campaign: International guidelines for management of severe sepsis 
and septic shock, 2012. Intensive Care Medicine, 39(2), 165-228. doi: 10.1007/s00134$012-2769-8$

Do, N. T., Ta, N. T., Tran, N. T., Than, H. M., Vu, B. T., Hoang, L. B., ... Wertheim, H. F. (2016). Point-of-care C-reactive protein testing to reduce inappropriate use of antibiotics for non-severe acute respiratory infections in Vietnamese primary health care: a randomised controlled trial. The Lancet. Global health, 4(9), e633-e641. doi:10.1016/S2214-109X(16)30142-5

Dragan, V., Wei, Y., Elligsen, M., Kiss, A., Walker, S. A. N., \& Leis, J. A. (2018). Prophylactic antimicrobial therapy for acute aspiration pneumonitis. Clinical Infectious Diseases : An Official Publication of the Infectious Diseases Society of America, 67(4), 513-518. doi: $10.1093 /$ cid/ciy120

Dulhunty, J. M., Roberts, J. A., Davis, J. S., Webb, S. A., Bellomo, R., Gomersall, C.,...BLING II Investigators for the ANZICS Clinical Trials Group .( 2015). A multicenter randomized trial of continuous versus intermittent beta-lactam infusion in severe sepsis. American Journal of Respiratory and Critical Care Medicine, 192(11), 1298-1305. doi: 10.1164/rccm.201505-0857OC

Filice, G. A., Drekonja, D. M., Thurn, J. R., Rector, T. S., Hamann, G. M., Masoud, B. T.,... Johnson JR. (2013). Use of a computer decision support system and antimicrobial therapy appropriateness. Infection Control and Hospital Epidemiology, 34(6), 558-565. doi: $10.1086 / 670627$

Filippini, M., Ortiz, L. G., \& Masiero, G. (2013). Assessing the impact of national antibiotic campaigns in Europe. The European Journal of Health Economics : HEPAC : Health Economics in Prevention and Care, 14(4), 587-599. doi: 10.1007/s10198-012-0404-9

Fisher, H., Oluboyede, Y., Chadwick, T., Abdel-Fattah, M., Brennand, C., Fader, M.,... Pickard R. (2018). Continuous low-dose antibiotic prophylaxis for adults with repeated 
urinary tract infections (AnTIC): A randomised, open-label trial. The Lancet.Infectious Diseases, 18(9), 957-968. doi: 10.1016/S1473-3099(18)30279-2

Garnacho-Montero, J., Escoresca-Ortega, A., \& Fernandez-Delgado, E. (2015). Antibiotic de-escalation in the ICU: How is it best done? Current Opinion in Infectious Diseases, 28(2), 193-198. doi: 10.1097/QCO.0000000000000141.

Grabe, M., Bartoletti, R., Bjerklund-Johansen, T. E., Cai, T., Çek, M., Köves, B.,...Wullt, B. Guidelines on urological infections. European Association of Urology Web site. Retrieved from: http://uroweb.org/wp-content/uploads/19-Urological-infections LR2.pdf.

Hermsen, E. D., VanSchooneveld, T. C., Sayles, H., \& Rupp, M. E. (2012). Implementation of a clinical decision support system for antimicrobial stewardship. Infection Control and Hospital Epidemiology, 33(4), 412-415. doi: 10.1086/664762

Herrmann, M. (2010). Monopoly pricing of an antibiotic subject to bacterial resistance. Journal of Health Economics, 29(1), 137-150. doi: 10.1016/j.jhealeco.2009.11.007

Hijazi, K., Mukhopadhya, I., Abbott, F., Milne, K., Al-Jabri, Z. J., Oggioni, M. R., et al. (2016). Susceptibility to chlorhexidine amongst multidrug-resistant clinical isolates of staphylococcus epidermidis from bloodstream infections. International Journal of Antimicrobial Agents, 48(1), 86-90. doi: 10.1016/j.jjantimicag.2016.04.015

Huang, S. S., Septimus, E., Kleinman, K., Moody, J., Hickok, J., Avery, T. R., ,. AHRQ DECIDE Network and Healthcare-Associated Infections Program. (2013). Targeted versus universal decolonization to prevent ICU infection. The New England Journal of Medicine, 368(24), 2255-2265. doi: 10.1056/NEJMoa1207290

Huijts, S. M., van Werkhoven, C. H., Boersma, W. G., Buijs, J., Buunk, G., Compaijen, C. J.,...Bonten MJ (2013). Guideline adherence for empirical treatment of pneumonia and patient outcome. treating pneumonia in the Netherlands. The Netherlands Journal of Medicine, 71(10), 502-507. http://www.nimonline.nl/ 
Infectious Diseases Society of America (IDSA), Spellberg, B., Blaser, M., Guidos, R. J., Boucher, H. W., Bradley, J. S.,...Gilbert DN. (2011). Combating antimicrobial resistance: Policy recommendations to save lives. Clinical Infectious Diseases : An Official Publication of the Infectious Diseases Society of America, 52 Suppl 5, S397428. doi: $10.1093 / \mathrm{cid} / \mathrm{cir} 153$.

Jamhour, A., El-Kheir, A., Salameh, P., Hanna, P. A., \& Mansour, H. (2017). Antibiotic knowledge and self-medication practices in a developing country: A cross-sectional study. American Journal of Infection Control, 45(4), 384-388. doi:

10.1016/j.ajic.2016.11.026

Just Say Sepsis! A review of the process of care received by patients with sepsis. A report by the National Confidential Enquiry into Patient Outcome and Death 2015. Retrieved from: https://www.ncepod.org.uk/2015report2/downloads/JustSaySepsis FullReport.pdf

Keitel, K., Kagoro, F., Samaka, J., Masimba, J., Said, Z., Temba, H., ... D'Acremont, V. (2017). A novel electronic algorithm using host biomarker point-of-care tests for the management of febrile illnesses in Tanzanian children (e-POCT): A randomized, controlled non-inferiority trial. PLoS medicine, 14(10), e1002411. doi:10.1371/journal.pmed.1002411

Kelly, R., Zoubiane, G., Walsh, D., Ward, R., \& Goossens, H. (2016). Public funding for research on antibacterial resistance in the JPIAMR countries, the European commission, and related European union agencies: A systematic observational analysis. The Lancet.Infectious Diseases, 16(4), 431-440. doi: 10.1016/S14733099(15)00350-3

Klein, E. Y., Van Boeckel, T. P., Martinez, E. M., Pant, S., Gandra, S., Levin, S. A.,...Laxminarayan R. (2018). Global increase and geographic convergence in antibiotic consumption between 2000 and 2015. Proceedings of the National Academy of 
Sciences of the United States of America, 115(15), E3463-E3470. doi: 10.1073/pnas.1717295115

Lawes, T., Lopez-Lozano, J. M., Nebot, C. A., Macartney, G., Subbarao-Sharma, R., Dare, C. R.,...Gould, I. M. (2015). Effects of national antibiotic stewardship and infection control strategies on hospital-associated and community-associated meticillin-resistant staphylococcus aureus infections across a region of Scotland: A non-linear time-series study. The Lancet.Infectious Diseases, 15(12), 1438-1449. doi: 10.1016/S14733099(15)00315-1

Lawes, T., Lopez-Lozano, J. M., Nebot, C. A., Macartney, G., Subbarao-Sharma, R., Wares, K. D.,...Gould, I. M. (2017). Effect of a national 4C antibiotic stewardship intervention on the clinical and molecular epidemiology of clostridium difficile infections in a region of Scotland: A non-linear time-series analysis. The Lancet.Infectious Diseases, 17(2), 194206. doi: 10.1016/S1473-3099(16)30397-8

Laxminarayan, R., Sridhar, D., Blaser, M., Wang, M., \& Woolhouse, M. (2016). Achieving global targets for antimicrobial resistance. Science (New York, N.Y.), 353(6302), 874875. doi: $10.1126 /$ science.aaf9286

Lee, C. R., Lee, J. H., Kang, L. W., Jeong, B. C., \& Lee, S. H. (2015). Educational effectiveness, target, and content for prudent antibiotic use. BioMed Research International, 2015, 214021. doi: 10.1155/2015/214021

Little, P., Stuart, B., Francis, N., Douglas, E., Tonkin-Crine, S., Anthierens, S.,...GRACE consortium.. (2013). Effects of internet-based training on antibiotic prescribing rates for acute respiratory-tract infections: A multinational, cluster, randomised, factorial, controlled trial. Lancet, 382(9899), 1175-1182. doi: 10.1016/S0140-6736(13)60994-0

Littmann, J., Buyx, A., \& Cars, O. (2015). Antibiotic resistance: An ethical challenge. International Journal of Antimicrobial Agents, 46(4), 359-361. doi: 10.1016/j.ijantimicag.2015.06.010 
López-Lozano, J-M., Lawes, T., Nebot C., Beyaert, A., Bertrand, X., Hocquet, D., ...THRESHOLDS study group. A nonlinear time-series analysis approach to identify thresholds in associations between population antibiotic use and rates of resistance. Nature Microbiology. Advance online publication. doi: 10.1038/s41564-019-0410-0. [Epub ahead of print]

Lu, Z., Chen, Y., Chen, W., Liu, H., Song, Q., Hu, X.,...Han L. (2015). Characteristics of qacA/B-positive staphylococcus aureus isolated from patients and a hospital environment in china. The Journal of Antimicrobial Chemotherapy, 70(3), 653-657. doi: 10.1093/jac/dku456

Magouras, I., Carmo, L. P., Stark, K. D. C., \& Schupbach-Regula, G. (2017). Antimicrobial usage and -resistance in livestock: Where should we focus? Frontiers in Veterinary Science, 4, 148. doi: 10.3389/fvets.2017.00148

Masterton, R. G. (2011). Antibiotic de-escalation. Critical Care Clinics, 27(1), 149-162. doi: 10.1016/j.ccc.2010.09.009.

Mc Cay, P. H., Ocampo-Sosa, A. A., \& Fleming, G. T. (2010). Effect of subinhibitory concentrations of benzalkonium chloride on the competitiveness of pseudomonas aeruginosa grown in continuous culture. Microbiology, 156(Pt 1), 30-38. doi: 10.1099/mic.0.029751-0

Morel, C. M., \& Mossialos, E. (2010). Stoking the antibiotic pipeline. BMJ (Clinical Research Ed.), 340, c2115. doi: 10.1136/bmj.c2115

Newland, J. G., Stach, L. M., De Lurgio, S. A., Hedican, E., Yu, D., Herigon, J. C., ... Zaoutis, T. E. (2012). Impact of a prospective-audit-with-feedback antimicrobial stewardship program at a children's hospital. Journal of the Pediatric Infectious Diseases Society, 1(3), 179-186. doi: 10.1093/jpids/pis054 
Niel-Weise, B. S., van den Broek, P. J., da Silva, E. M., \& Silva, L. A. (2012). Urinary catheter policies for long-term bladder drainage. The Cochrane Database of Systematic Reviews, (8):CD004201. doi: 10.1002/14651858.CD004201.pub3.

Oggioni, M. R., Coelho, J. R., Furi, L., Knight, D. R., Viti, C., Orefici, G.,...BIOHYPO consortium. (2015). Significant differences characterise the correlation coefficients between biocide and antibiotic susceptibility profiles in staphylococcus aureus. Current Pharmaceutical Design, 21(16), 2054-2057. doi:

$10.2174 / 1381612821666150310103238$

Ohji, G., Doi, A., Yamamoto, S., \& Iwata, K. (2016). Is de-escalation of antimicrobials effective? A systematic review and meta-analysis. International Journal of Infectious Diseases : IJID : Official Publication of the International Society for Infectious Diseases, 49, 71-79. doi: 10.1016/j.jijid.2016.06.002

Otter, J. A., Patel, A., Cliff, P. R., Halligan, E. P., Tosas, O., \& Edgeworth, J. D. (2013). Selection for qacA carriage in CC22, but not CC30, methicillin-resistant staphylococcus aureus bloodstream infection isolates during a successful institutional infection control programme. The Journal of Antimicrobial Chemotherapy, 68(5), 992-999. doi: $10.1093 / \mathrm{jac} / \mathrm{dks} 500$

One Health Basics. Retrieved from: https://www.cdc.gov/onehealth/basics/index.html

O'Neill J. Review on Antimicrobial Resistance Antimicrobial Resistance: Tackling a crisis for the health and wealth of nations. London: Review on Antimicrobial Resistance. 2014. Retrieved from: https://amrreview.org/sites/default/files/AMR\%20Review\%20Paper\%20\%20Tackling\%20a\%20crisis\%20for\%20the\%20health\%20and\%20wealth\%20of\%20nati ons 1.pdf

Oonsivilai, M., Mo, Y., Luangasanatip, N., Lubell, Y., Miliya, T., Tan, P., ... Cooper, B. S. (2018). Using machine learning to guide targeted and locally-tailored empiric antibiotic 
prescribing in a children's hospital in Cambodia. Wellcome open research, 3, 131. doi:10.12688/wellcomeopenres.14847.1

Organization, W.H., WHO updates Essential Medicines List with new advice on use of antibiotics, and adds medicines for hepatitis C, HIV, tuberculosis and cancer.2017. Retrieved from: https://www.who.int/news-room/detail/06-06-2017-who-updatesessential-medicines-list-with-new-advice-on-use-of-antibiotics-and-adds-medicines-forhepatitis-c-hiv-tuberculosis-and-cancer

Ozawa, S., Evans, D. R., Bessias, S., Haynie, D. G., Yemeke, T. T., Laing, S. K., \& Herrington, J. E. (2018). Prevalence and estimated economic burden of substandard and falsified medicines in low- and middle-income countries: A systematic review and meta-analysis. JAMA Network Open, 1(4), e181662. doi:

10.1001/jamanetworkopen.2018.1662

Parsonage, B., Hagglund, P. K., Keogh, L., Wheelhouse, N., Brown, R. E., \& Dancer, S. J. (2017). Control of antimicrobial resistance requires an ethical approach. Frontiers in Microbiology, 8, 2124. doi: 10.3389/fmicb.2017.02124

Paul, M., Dickstein, Y., \& Raz-Pasteur, A. (2016). Antibiotic de-escalation for bloodstream infections and pneumonia: Systematic review and meta-analysis. Clinical Microbiology and Infection : The Official Publication of the European Society of Clinical Microbiology and Infectious Diseases, 22(12), 960-967. doi: 10.1016/j.cmi.2016.05.023

Peterson, L. R. (2005). Squeezing the antibiotic balloon: The impact of antimicrobial classes on emerging resistance. Clinical Microbiology and Infection: The Official Publication of the European Society of Clinical Microbiology and Infectious Diseases, 11 Suppl 5, 416. doi: 10.1111/j.1469-0691.2005.01238.x

Pouwels, K. B., Hopkins, S., Llewelyn, M. J., Walker, A. S., McNulty, C. A., \& Robotham, J. V. (2019). Duration of antibiotic treatment for common infections in English primary 
care: cross sectional analysis and comparison with guidelines. BMJ (Clinical research ed.), 364, 1440. doi:10.1136/bmj.1440

Pradelli, J., Risso, K., de Salvador, F. G., Cua, E., Ruimy, R., \& Roger, P. M. (2015). Community-acquired pneumonia: Impact of empirical antibiotic therapy without respiratory fluoroquinolones nor third-generation cephalosporins. European Journal of Clinical Microbiology \& Infectious Diseases : Official Publication of the European Society of Clinical Microbiology, 34(3), 511-518. doi: 10.1007/s10096-014-2254-0

Pulido, M. R., Garcia-Quintanilla, M., Martin-Pena, R., Cisneros, J. M., \& McConnell, M. J. (2013). Progress on the development of rapid methods for antimicrobial susceptibility testing. The Journal of Antimicrobial Chemotherapy, 68(12), 2710-2717. doi: 10.1093/jac/dkt253

Quenot, J. P., Luyt, C. E., Roche, N., Chalumeau, M., Charles, P. E., Claessens, Y. E.,...Brun-Buisson C. (2013). Role of biomarkers in the management of antibiotic therapy: An expert panel review II: Clinical use of biomarkers for initiation or discontinuation of antibiotic therapy. Annals of Intensive Care, 3(1), 21-5820-3-21. doi: $10.1186 / 2110-5820-3-21$

Ranji, S. R., Steinman, M. A., Shojania, K. G., \& Gonzales, R. (2008). Interventions to reduce unnecessary antibiotic prescribing: A systematic review and quantitative analysis. Medical Care, 46(8), 847-862. doi: 10.1097/MLR.0b013e318178eabd

Rondeau, C., Chevet, G., Blanc, D. S., Gbaguidi-Haore, H., Decalonne, M., Dos Santos, S.,... van der Mee-Marquet NI. (2016). Current molecular epidemiology of methicillinresistant staphylococcus aureus in elderly French people: Troublesome clones on the horizon. Frontiers in Microbiology, 7, 31. doi: 10.3389/fmicb.2016.00031

Report by the Director-General-WHO. Draft thirteenth general programme of work, 20192023. Retrieved from: http://apps.who.int/gb/ebwha/pdf files/WHA71/A71 4en.pdf?ua=1 
Resistance Map. Center for Disease Dynamics, Economics \& Policy (2017). Retrieved from: https://resistancemap.cddep.org/

Rzewuska, M., Charani, E., Clarkson, J. E., Davey, P. G., Duncan, E. M., Francis, J. J.,...Joint Programming Initiative on Antimicrobial Resistance (JPIAMR) Working Group on Behavioural Approaches to Antibiotic Stewardship Programs.. (2019). Prioritizing research areas for antibiotic stewardship programmes in hospitals: A behavioural perspective consensus paper. Clinical Microbiology and Infection : The Official Publication of the European Society of Clinical Microbiology and Infectious Diseases, 25(2), 163-168. doi: 10.1016/j.cmi.2018.08.020

Selekman, R. E., Shapiro, D. J., Boscardin, J., Williams, G., Craig, J. C., Brandstrom, P., ... Copp, H. L. (2018). Uropathogen resistance and antibiotic prophylaxis: A meta-analysis. Pediatrics, 142(1), pii: e20180119. doi: 10.1542/peds.2018-0119

Schuts, E. C., Hulscher, M. E. J. L., Mouton, J. W., Verduin, C. M., Stuart, J. W. T. C., Overdiek, H. W. P. M.,...Prins, J. M. (2016). Current evidence on hospital antimicrobial stewardship objectives: A systematic review and meta-analysis. The Lancet.Infectious Diseases, 16(7), 847-856. doi: 10.1016/S1473-3099(16)00065-7

Shiu, J., Wang, E., Tejani, A. M., \& Wasdell, M. (2013). Continuous versus intermittent infusions of antibiotics for the treatment of severe acute infections. The Cochrane Database of Systematic Reviews, 3, CD008481. doi:

10.1002/14651858.CD008481.pub2

Silva, B. N., Andriolo, R. B., Atallah, A. N., \& Salomao, R. (2013). De-escalation of antimicrobial treatment for adults with sepsis, severe sepsis or septic shock. The Cochrane Database of Systematic Reviews, 3, CD007934. doi: 10.1002/14651858.CD007934.pub3

Sirijatuphat, R., Sripanidkulchai, K., Boonyasiri, A., Rattanaumpawan, P., Supapueng, O., Kiratisin, P.,...Thamlikitkul V. (2018). Implementation of global antimicrobial resistance 
surveillance system (GLASS) in patients with bacteremia. PloS One, 13(1), e0190132. doi: 10.1371/journal.pone.0190132

Teshome, B. F., Vouri, S. M., Hampton, N., Kollef, M. H., \& Micek, S. T. (2019). Duration of exposure to antipseudomonal beta-lactam antibiotics in the critically ill and development of new resistance. Pharmacotherapy, 39(3), 261-270. doi: 10.1002/phar.2201

Tackling antimicrobial resistance (AMR) together. Working paper 1.0: Multisectoral coordination. Geneva: World Health Organization (2018) (WHO/HWSI/AMR/2018.2). Retrieved from: https://www.who.int/antimicrobial-resistance/national-actionplans/AMRGenderEquityGuidance-Sept2018.pdf?ua=1

Takesue, Y., Nakajima, K., Ichiki, K., Ishihara, M., Wada, Y., Takahashi, Y.,...lkeuchi H. (2010). Impact of a hospital-wide programme of heterogeneous antibiotic use on the development of antibiotic-resistant gram-negative bacteria. The Journal of Hospital Infection, 75(1), 28-32. doi: 10.1016/j.jhin.2009.11.022

Tang, K. L., Caffrey, N. P., Nóbrega, D. B., Cork, S. C., Ronksley, P. E., Barkema, H. W.,... Ghali, W. A. (2017). Restricting the use of antibiotics in food-producing animals and its associations with antibiotic resistance in food-producing animals and human beings: $\mathrm{A}$ systematic review and meta-analysis. Lancet Planet Health, 1(8), e316-e327. doi: $10.1016 / \mathrm{S} 2542-5196(17) 30141-9$

The UK's five-year national action plan. Tackling antimicrobial resistance 2019-2024. Retrieved from: https://assets.publishing.service.gov.uk/government/uploads/system/uploads/attachmen $\underline{\mathrm{t} \text { data/file/773130/uk-amr-5-year-national-action-plan.pdf }}$

Thomas, Z., Bandali, F., Sankaranarayanan, J., Reardon, T., Olsen, K. M., \& Critical Care Pharmacotherapy Trials Network. (2015). A multicenter evaluation of prolonged empiric antibiotic therapy in adult ICUs in the united states. Critical Care Medicine, 43(12), 2527-2534. doi: 10.1097/CCM.0000000000001294 
Tiong, J. J., Loo, J. S., \& Mai, C. W. (2016). Global antimicrobial stewardship: A closer look at the formidable implementation challenges. Frontiers in Microbiology, 7, 1860. doi: 10.3389/fmicb.2016.01860

Toth, H., Fesus, A., Kungler-Goracz, O., Balazs, B., Majoros, L., Szarka, K., Kardos, G. (2018). Utilization of vector autoregressive and linear transfer models to follow up the antibiotic resistance spiral in gram-negative bacteria from cephalosporin consumption to colistin resistance. Clinical Infectious Diseases : An Official Publication of the Infectious Diseases Society of America, Advance online publication. doi: 10.1093/cid/ciy1086. [Epub ahead of print]

UNEP (2019). Frontiers 2018/19 Emerging Issues of Environmental Concern. United Nations Environment Programme, Nairobi. Retrieved from: https://wedocs.unep.org/bitstream/handle/20.500.11822/27538/Frontiers1819.pdf?sequ ence $=1$ \&isAllowed $=y$

van Belkum, A., \& Dunne, W. M.,Jr. (2013). Next-generation antimicrobial susceptibility testing. Journal of Clinical Microbiology, 51(7), 2018-2024. doi: 10.1128/JCM.00313-13

van Duijn, P. J., \& Bonten, M. J. (2014). Antibiotic rotation strategies to reduce antimicrobial resistance in Gram-negative bacteria in European intensive care units: study protocol for a cluster-randomized crossover controlled trial. Trials, 15, 277. doi:10.1186/17456215-15-277

Vanderhaeghen, W., \& Dewulf, J. (2017). Antimicrobial use and resistance in animals and human beings. The Lancet.Planetary Health, 1(8), e307-e308. doi: 10.1016/S25425196(17)30142-0

Wand, M. E., Bock, L. J., Bonney, L. C., \& Sutton, J. M. (2016). Mechanisms of increased resistance to chlorhexidine and cross-resistance to colistin following exposure of klebsiella pneumoniae clinical isolates to chlorhexidine. Antimicrobial Agents and Chemotherapy, 61(1), pii: e01162-16. doi: 10.1128/AAC.01162-16 
Webber, M. A., Whitehead, R. N., Mount, M., Loman, N. J., Pallen, M. J., \& Piddock, L. J. (2015). Parallel evolutionary pathways to antibiotic resistance selected by biocide exposure. The Journal of Antimicrobial Chemotherapy, 70(8), 2241-2248.

WebResist (version 2.0) [Computer software]. Location: http://www.webresist.org/

WHO. Global Action Plan on Antimicrobial Resistance. World Health Organization, Geneva (2015). Retrieved from:

http://www.wpro.who.int/entity/drug resistance/resources/global action plan eng.pdf

World Bank. 2017. "Drug-Resistant Infections: A Threat to Our Economic Future". Washington, DC: World Bank. Retrieved from:

http://www.worldbank.org/en/topic/health/publication/drug-resistant-infections-a-threatto-our-economic-future

Yeo, C. L., Chan, D. S., Earnest, A., Wu, T. S., Yeoh, S. F., Lim, R.,...Hsu, L. Y. (2012). Prospective audit and feedback on antibiotic prescription in an adult hematologyoncology unit in Singapore. European Journal of Clinical Microbiology \& Infectious Diseases : Official Publication of the European Society of Clinical Microbiology, 31(4), 583-590. doi: 10.1007/s10096-011-1351-6

Yong, M. K., Buising, K. L., Cheng, A. C., \& Thursky, K. A. (2010). Improved susceptibility of gram-negative bacteria in an intensive care unit following implementation of a computerized antibiotic decision support system. The Journal of Antimicrobial Chemotherapy, 65(5), 1062-1069. doi: 10.1093/jac/dkq058

Zamudio, R., Oggioni, M. R., Gould, I. M., \& Hijazi, K. (2019). Time for biocide stewardship? Nature Microbiology, Advance online publication. doi: 10.1038/s41564-019-0360-6. [Epub ahead of print] 